\title{
General dental practitioners' perceptions of antimicrobial use and resistance: a qualitative interview study
}
IN BRIEF
- Provides insights into beliefs and attitudes held by general dental practitioners (GDPs) with regard to antibiotic use and resistance.
- Reports that perceptions of resistance may vary between GDPs.
- Suggests that antibiotic prescribing practices of GDPs may be influenced by both immediate clinical pressures and longer term public health concerns.

\author{
A. L. Cope, ${ }^{* 1}$ F. Wood, ${ }^{2}$ N. A. Francis ${ }^{3}$ and I. G. Chestnutt ${ }^{4}$
}

Background Dentists are responsible for 9-10\% of all antibiotics dispensed in primary care in the UK, many of which may be provided contrary to clinical guidelines. Since antibiotic consumption has been identified as a major cause of antibiotic resistance, dental prescribing may be a significant contributor to this important public health problem. Objective This study aims to explore general dental practitioners' (GDPs) perceptions and attitudes towards antibiotic use and resistance. Method Qualitative interview study with 19 purposively sampled GDPs working in Wales. A set of open-ended questions were developed and amended during semi-structured telephone interviews. Interviews were recorded, transcribed verbatim and codes were developed using thematic analysis. Results Perceptions of antibiotic use and resistance varied widely between practitioners, particularly with respect to the prevalence and impact of resistant strains on the management of dentoalveolar infection, and the impact of dental prescribing on the emergence of resistance. GDPs reported that their antibiotic prescribing decisions were driven by both clinical pressures and wider public health considerations. Conclusions Interventions to enhance the quality of antibiotic prescribing in primary care dentistry should address issues associated with inappropriate prescribing as well as providing education about the causes, prevalence and impact of antibiotic resistance.

\section{INTRODUCTION}

Since their introduction in the 1930s and 1940s, antibiotics have saved countless lives. However, soon after their earliest trials it became evident that some bacteria possessed, or could acquire, resistance to these agents. During the intervening 80 years there has been a gradual and sustained emergence of antimicrobial resistant bacterial strains, while the number of new antibiotics has decreased, leading to widespread concern about the impact antibiotic resistance could have on the management of infectious diseases. ${ }^{1-3}$

Antibiotic resistance is considered a pressing international public health problem, ${ }^{1,2}$ and is associated with increased morbidity, mortality and healthcare costs. ${ }^{4}$ Antibiotic consumption is recognised as a major cause of emerging resistance and some of the

'President's Research Scholar, ${ }^{2}$ Senior Lecturer, ${ }^{3}$ Senior Clinical Fellow, Cochrane Institute of Primary Care and Public Health, School of Medicine, Cardiff University, Third Floor, Neuadd Meirionnydd, Heath Park, Cardiff, Wales, CF14 4YS; ${ }^{4}$ Professor and Honorary Consultant in Dental Public Health, Applied Clinical Research and Public Health, Cardiff University Dental School, School of Dentistry, College of Biomedical and Life Sciences, Heath Park, Cardiff, Wales, CF14 4XY

*Correspondence to: Ms Anwen L. Cope

Email: copea1@cardiff.ac.uk; Tel: +44 (0) 2920687157

Online article number E9

Refereed Paper - accepted 20 May 2014

DOI: 10.1038/sj.bdj.2014.761

${ }^{\circ}$ British Dental Journal 2014; 217: E9 increase in resistance is directly attributed to the indiscriminate or poor use of antibiotics. ${ }^{5,6}$ In response, initiatives at local, national, and international levels have attempted to promote antibiotic stewardship among healthcare professionals. ${ }^{1,6}$ Many interventions to improve prescribing occur within primary care, as approximately $80 \%$ of all antibiotics are prescribed within this setting. ${ }^{7}$ Despite this, qualitative research has suggested that many general practitioners (GPs) working in primary care do not perceive antibiotic resistance as a problem in their practice, but rather regard it as a secondary care or hospital problem..$^{8,9}$

Dentists currently prescribe approximately 9-10\% of all antibiotics dispensed in primary care in the United Kingdom, ${ }^{10,11}$ and therefore the possible contribution of dental prescribing to the development of antibiotic resistance should not be underestimated. There is concern that antibiotics are frequently prescribed inappropriately in the management of acute dental conditions where they may be of little clinical benefit, such as in the treatment of irreversible pulpitis, or as a substitute to providing local, operative measures or adequate analgesia. ${ }^{12,13}$ In addition, antibiotic therapy prescribed by dentists is typically empirical, employing broad-spectrum agents that can predispose to the selection of resistant strains. ${ }^{14}$ Antibiotic resistant bacteria are increasingly isolated from odontogenic infections. ${ }^{15-18}$ Furthermore, studies have identified a correlation between the emergence of antibiotic resistant bacteria such as Prevotella $s p$. and previous administration of antibiotics for dental infections. ${ }^{18}$

While a previous study has examined the antibiotic prescribing knowledge among dental professionals, ${ }^{19}$ to date there has been no research undertaken into GDPs' perceptions of antibiotic resistance. Therefore the aim of the study reported here was to explore clinicians' beliefs and attitudes towards antibiotic use and resistance.

\section{METHODS}

Qualitative methods were selected as being the most appropriate for exploring practitioners' perceptions of antibiotic resistance. Such methods do not seek to generate statistically representative data, instead they endeavour to interpret, or obtain a 'deeper understanding' of certain aspects of human beliefs, attitudes or behaviour. ${ }^{20}$ Since little is currently known about attitudes of members of the dental profession towards antibiotic resistance, a qualitative approach allowed researchers to have greater freedom to explore issues and, if necessary, to enquire about tangential topics that had not previously been considered. This contrasts 
with quantitative approaches with structured questionnaires that may be driven by researcher's preconceptions.

\section{Subjects and sampling}

This analysis was completed as part of a larger study examining influences on antibiotic prescribing behaviours of GDPs. We interviewed a 'purposive sample' of clinicians who had taken part in a cross-sectional study investigating the surgical and pharmacological management of adults with acute dental conditions within the General Dental Service (the Antibiotics and Primary Care Dental Problems [APICAL] study). The 42 GDPs participating in APICAL had been randomly selected from publically available databases of GDPs providing state-subsidised National Health Service (NHS) or private dental treatment to adult patients in Wales.

From those GDPs who expressed a willingness to take part in an interview, participants were selected on the basis of their location and length of time since qualification. This was done to obtain maximum variation within the sample. Non-probabilistic sampling techniques were employed as the objectives of the interviews were not to obtain a representative sample but to explore a wide range of perspectives and experience among GDPs.

The study was reviewed and given a favourable ethical opinion by the London Central Proportionate Review Committee (12/L0/1213). All GDPs provided their written consent before interview.

\section{Data collection}

Semi-structured telephone interviews were conducted with participating clinicians by a researcher trained in qualitative interviewing techniques (AC). AC is a qualified dentist undertaking postgraduate research and at the time when the interviews were conducted (July - October 2013) was a practicing GDP. All participants were made aware of her professional identity before the interview.

An interview guide was prepared before data collection. This was informed by the scientific literature, topic guides from other studies and clinical experiences of the research team. In the early stages of interviewing, the topic guide went through several iterations during which questions were added and revised.

Before the interview it was stressed to participants that the goal of the interviews were not to test knowledge but to understand the practitioner's experiences and point of view.

All interviews were audio recorded and transcribed verbatim, apart from one interview in which the recording device failed. No notes were recorded during the interview other than memos of additional questions and probes to ask each participant, to encourage active listening on the part of the interviewer. Instead, field notes were recorded in the form of a 'qualitative diary' following each interview. Interviews were anonymised on transcription and checked against the original recording to ensure fidelity, this also ensured the researcher (AC) was immersed in the dataset.

\section{Analysis}

Thematic analysis techniques as described by Braun and Clarke were employed. ${ }^{21}$ Transcripts were first coded to identify different features of the dataset with codes representing "the most basic segment, or element, of the raw data or information.22 A second qualitative researcher (FW) then second coded $20 \%$ of the transcripts to examine the coding system. Codes were then collated, defined and grouped into themes. Themes were then reviewed, defined and named, before all data coded as 'antibiotic resistance' were extracted for further analysis for the purposes of this paper. Analysis was facilitated by the qualitative data analysis software package NVivo (QSR International). ${ }^{23}$

Data collection, transcription and analysis was undertaken concurrently to examine the emergence of themes and at which point 'saturation' had been reached. Saturation is defined as 'data adequacy' and in practice is the process of collecting data until no new information is obtained. ${ }^{24}$ Since there are no published guidelines regarding how to assess data saturation in qualitative research, within this study saturation was judged to be when the richness of data within a theme no longer appeared to be increasing with subsequent interviews. Therefore the interview process ceased when the researcher felt that there was sufficient data to build a comprehensive and convincing insight into GDP behaviour. This point was judged to have been following coding of the nineteenth interview.

\section{RESULTS}

Out of the 27 GDPs who expressed an interest in participating in an interview, 19 were interviewed. Out of the 19 participating clinicians, 10 were male and the median number of years since graduation was 17. All but one qualified from dental schools in the UK and five had postgraduate qualifications such as MJDF/MFDS or MSc. Just over half (10/19) worked in practices in urban locations with the remainder based in town and fringe locations. Practitioners were based throughout all seven Welsh Local Health Boards (LHBs). While most practitioners undertook both NHS and private work, 15 reported that they spent the majority of their time treating patients under the NHS. Thirteen practitioners worked in locations that had Wales Index of Multiple Deprivation (WIMD) 2011 a ranks, the official measure of relative deprivation for small areas in Wales, ${ }^{25}$ indicating they served the $50 \%$ most deprived populations, while six were from practices that had WIMD scores suggesting they served the 50\% least deprived areas. Interviews lasted on average 25.6 minutes (SD 4.93 minutes).

\section{Awareness of resistance}

All clinicians were aware of antibiotic resistance, with many citing the example of methicillin-resistant Staphylococcus aureus (MRSA). There was an appreciation that prevalence of resistance was increasing and that this may present therapeutic problems for secondary care practitioners should individuals harbouring resistance contract a 'major' infection. Practitioners' knowledge of antibiotic resistance was informed by both 'formal' sources such as undergraduate teaching, postgraduate courses and journal articles, as well as media reports.

There were conflicting opinions as to whether antibiotic resistance occurred within dentoalveolar infections. Most of the clinicians interviewed had experienced antibiotic treatment failure, and some attributed this to the presence of resistant bacteria, particularly in relation to amoxicillin. However, other practitioners attributed antibiotic failure to achieve adequate pus drainage and emphasised the need to perform local measures in addition to prescribing antibiotics in situations of dentoalveolar infections.

'I would say 20 years ago amoxicillin seemed to clear most swollen faces quite quickly erm, now I'm finding amoxicillin, it might work, but I'm using metronidazole and amoxicillin more often, in combination... I definitely worry more about a severe infection and a lot aren't responding to amoxicillin and that's the first choice.' (GDP23, $>30$ years since qualification)

\section{Causes of resistance}

All dentists identified the link between antimicrobial prescribing and increasing prevalence of resistance. While many practitioners described how overprescribing could contribute to antibiotic resistance, there were varying levels of understanding about how antibiotic prescribing could lead to the emergence of resistant strains.

'...some of them [bacteria] will occasionally mutate, so what that means is that their, sort of their DNA, their genetic makeup like we have, changes a bit. Sometimes these changes mean that the antibiotics that we can give you won't work. And sometimes 
when antibiotics are given for the wrong reasons you can end up leaving some of these bacteria around, which then mutate, which then change, which means that the next time we maybe give antibiotics the bacteria that mutated, that changed, are still with us.' (GDP5, <10 years since qualification)

'(Laughing) I don't know too much about it to be honest.' (GDP24, <10 years since qualification)

Clinicians' perceptions of the extent to which prescribing by the dental profession contributed to antibiotic resistance varied between individuals. However, the majority of practitioners believed that while all antibiotic prescribing could theoretically lead to the emergence of resistance, the contribution of dental prescribing to antibiotic resistance was likely to be far less than medical colleagues. Clinicians who reported that dental prescribing was likely to have little to no impact on the prevalence of antibiotic resistance generally justified this due to the fewer number, shorter courses and narrower range of antibiotics dentists are able to prescribe in comparison with other medical professionals.

Several practitioners specifically cited overprescribing by general medical practitioners (GMPs) as the most significant cause of antibiotic resistance. Other explanations offered for antibiotic resistance included antibiotic use in agriculture and farming, prescribing by hospital doctors and the availability of over the counter antibiotics in developing countries.

'The dental team, compared to our medical cousins, we actually prescribe very, very little, compared to them... we can't bear the brunt, as it is, of antibiotic resistance developing in the world.' (GDP8, >30 years since qualification)

'I know some people who've got a [medical] problem and their GP will end up giving them 7 days of something and then another 7 days and then another 7 days. I think that's probably going to cause more problems than a 4 day course from the dentist'. (GDP6, $<10$ years since qualification)

\section{Responding to resistance}

Some practitioners, particularly those who had been qualified in excess of 20 years, discussed changing their prescribing patterns either directly or indirectly because of antibiotic resistance.

'...though I will try not to prescribe. I am, I am, I think we're all very aware, I mean 25 years ago we were probably prescribing, maybe over prescribing, but today I think we're all very aware of all these resistant strains of bacteria and we do try not to prescribe. Well I do anyway' (GDP26, 20-30 years since qualification)
Several practitioners who had changed their prescribing behaviours had been partly influenced to do so because of the publication of clinical guidelines.

'I would say under current guidelines we really are trying to avoid giving antibiotics. There has been a big change, I mean in the timespan that we've worked in general practice there's been a huge change in that'. (GDP3, 20-30 years since qualification)

However, clinicians also acknowledged the difficulties balancing between clinical pressures and public health considerations.

'I'm becoming, obviously like a lot of practitioners, far more aware of the use of antibiotics now, and the misuse of antibiotics, so I am trying to prescribe less than I used to. They're the only option sometimes when you've got patients sitting on the shelves in the waiting room and you want to get home, and the nurse wants to go home, the easiest option is to put a prescription and bring them back a day or so later, but I would certainly never do that anymore.' (GDP37, $>30$ years since qualification)

'It really depends whether that patient presented at a time when I had a fair bit of time on my hands. Now if I was under pressure, I will be honest and tell you that I will write them a prescription for some antibiotics, if I've got six people sitting waiting for treatment, you know.' (GDP26, 20-30 years since qualification)

When asked what should be done to optimise prescribing among the dental profession, practitioners who primarily provided NHS services were more likely to suggest that feedback about current prescribing patterns and greater incentives to provide operative treatment for the management of acute dental conditions may encourage practitioners to prescribe fewer antibiotics. However, some GDPs, particularly those primarily providing private dentistry, described how raising awareness of resistance and appropriate management of acute dental conditions (for example, operative treatment) among the public would reduce patients' expectation of antibiotics and increase willingness to receive operative treatment.

\section{DISCUSSION}

This is the first qualitative study to explore GDPs' observation and understanding of antimicrobial resistance. We found that perceptions of resistance varied widely between practitioners, particularly with respect to the prevalence and impact of resistant strains on the management of dentoalveolar infections and the contribution of dental prescribing to emergence of resistance. Some practitioners felt strongly that they had experienced treatment failure directly related to antibiotic resistance, while others related poor clinical outcomes to inadequate surgical intervention. Many practitioners felt that prescribing practices of the dentists had improved during the last two decades, with several relating this change to the publication of clinical guidelines regarding antibiotic prescribing. Some GDPs felt that antibiotic prescribing of the dental profession could be improved if operative treatment for acute dental conditions was incentivised under the NHS contract while others described how increasing public awareness of resistance would reduce patient expectation of antibiotics and increase acceptance of operative treatment.

The GDPs interviewed were drawn from a wide range of geographical areas, served local communities with a range of deprivation and were a mix of private and NHS providers and performers. This allowed investigators to obtain a wide range of views and beliefs. However, clinicians who agreed to take part in APICAL may have had a special interest in antibiotic prescribing or postgraduate education. It is possible that we missed important data obtainable only from GDPs who did not wish to participate. While we attempted to interview practitioners from a broad purposive sample of GDPs, our aim was to identify important themes regarding antibiotic resistance, not to generate statements generalisable to other dental practitioners.

Interviewing GDPs about prescribing and antibiotic resistance was potentially professionally sensitive. Practitioners may be aware that their prescribing behaviour deviates from an 'ideal' that exists within clinical guidelines and therefore practitioners' responses may be biased to attempt to provide 'correct', rather than honest responses. However, all GDPs were reassured at the start of the interview that they were not going to be judged on their prescribing decisions and that no consequences would result from anything revealed during the study. In fact, investigators found that GDPs were typically candid about their usual practice and the pressures they experienced. This could be in part, attributable to the shared professional identity between the interviewer and participants, although similar openness has been described in studies where interviewers did not share the profession of the participants being interviewed. ${ }^{8}$

Similar to the current findings, a grounded theory study of GMPs' perceptions of antimicrobial resistance reported that clinicians' antibiotic prescribing decisions were a balancing act between immediate duty to their patient, patient pressure and wider responsibilities to public health. ${ }^{8}$ Dentists participating in the current study particularly highlighted the impact clinical 
time pressures could have on their decision to prescribe antibiotics for a patient with an acute dental condition. While the possible association between clinical time pressures and antibiotic prescribing habits of GDPs have been described previously, ${ }^{26}$ it still remains unclear the exact degree to which time pressures influence prescribing behaviours.

There was a belief among many of the GDPs interviewed that antibiotic prescribing behaviours of the dental profession as a whole had improved over the last few decades. However, recent studies have revealed that antibiotics are still widely used by dentists in the management of acute dental conditions, often without conjunctive local measures (such as dental extraction, endodontic treatment or incision and drainage of a swelling). ${ }^{12}$ This suggests that further efforts are needed to continue to impress upon dental professionals the importance of optimising antibiotic usage. Previous interventions that have successfully improved prescribing behaviour among GDPs have included clinical audit, ${ }^{27,28}$ and pharmacist delivered academic detailing. ${ }^{29}$ However, it is unclear whether these interventions result in long-term changes in antibiotic use and therefore further studies are required to identify the interventions that produce sustained improvements in prescribing behaviour. The current study also highlighted that some GDPs have deficiencies in their knowledge regarding the aetiology of antibiotic resistance, and are unsure about the impact dental prescribing could have on the emergence of resistance. Raising awareness of such matters within the dental community may positively influence some practitioners to improve their prescribing practice. Interventions will also need to take into account the influence clinical pressures have on antibiotic prescribing decisions in primary dental care.

\section{CONCLUSIONS}

This is the first study to provide insights into GDPs' perceptions of antibiotic resistance. Dental practitioners are aware of resistance but vary in their assessment of the impact of dental prescribing on the emergence of resistance. Antibiotic prescribing decisions taken by GDPs are complex and may be influenced by clinical pressures. Interventions to enhance the quality of antibiotic prescribing in primary care dentistry should address these issues while educating GDPs about the aetiology, prevalence and impact of antibiotic resistance.

The work was supported by: the Wales School for Primary Care Research (grant number 504,746); a President's Research Scholarship from Cardiff University (grant number RCUCO99). The authors declare no conflicts of interest with respect to the authorship and/or publication of this article.

1. Davies S C. Annual report of the chief medical officer, volume two, 2011. Infections and the rise of antimicrobial resistance. London: Department of Health, 2013.

2. World Health Organization. World Health Organization report on infectious diseases 2000: overcoming antimicrobial resistance. Geneva: WHO, 2000.

3. Lewis M A. Why we must reduce dental prescription of antibiotics: European Union Antibiotic Awareness Day. Br Dent J 2008; 205: 537-538.

4. Cosgrove S E. The relationship between antimicrobial resistance and patient outcomes: mortality, length of hospital stay, and health care costs. Clin Infect Dis 2006; 42: S82-S9.

5. Goossens $H_{1}$ Ferech M, Vander Stichele R, Elseviers M. Outpatient antibiotic use in Europe and association with resistance: a cross-national database study. Lancet 2005; 365: 579-587.

6. Costelloe C, Metcalfe C, Lovering A, Mant D, Hay A. Effect of antibiotic prescribing in primary care on antimicrobial resistance in individual patients: systematic review and meta-analysis. BMJ 2010; 340: c2096.

7. European Centre for Disease Prevention and Control (ECDC), European Medicines Agency (EMEA). The bacterial challenge: time to react. Stockholm: ECDC, 2009. Online article available at http://www.ecdc. europa.eu/en/publications/Publications/0909_ TER_The_Bacterial_Challenge_Time_to_React.pdf (accessed June 2014)

8. Simpson S A, Wood F, Butler C C. General practitioners' perceptions of antimicrobial resistance: a qualitative study. J Antimicrob Chemother 2007; 59: 292-296.

9. Wood F, Phillips C, Brookes-Howell L et al. Primary care clinicians' perceptions of antibiotic resistance: a multi-country qualitative interview study. J Antimicrob Chemother 2013; 68: 237-243.

10. Karki A J, Holyfield G, Thomas D. Dental prescribing in Wales and associated public health issues. Br Dent J 2011; 210: E21.

11. Prescribing and Primary Care Services. Prescribing by dentists: England 2012. London: Health and Social Care Information Centre, 2013

12. Tulip D E, Palmer N O. A retrospective investigation of the clinical management of patients attending an out of hours dental clinic in Merseyside under the new NHS dental contract. Br Dent J 2008; 205: 659-664.
13. Dailey Y M, Martin M V. Are antibiotics being used appropriately for emergency dental treatment? Br Dent J 2001; 191: 391-393.

14. Sweeney L C, Dave J, Chambers P A, Heritage J. Antibiotic resistance in general dental practice-a cause for concern? J Antimicrob Chemother 2004 53: 567-576.

15. Kuriyama T, Karasawa T, Nakagawa K, Nakamura $S$, Yamamoto E. Antimicrobial susceptibility of major pathogens of orofacial odontogenic infections to 11 $\beta$-lactam antibiotics. Oral Microbiol Immunol 2002; 17: 285-289.

16. Eick S, Pfister W, Straube E. Antimicrobial susceptibility of anaerobic and capnophilic bacteria isolated from odontogenic abscesses and rapidly progressive periodontitis. Int J Antimicrob Ag 1999; 12: $41-46$.

17. Teng $L J$, Hsueh $P R$, Chen $Y C$, Ho S W, Luh K T. Antimicrobial susceptibility of viridans group streptococci in Taiwan with an emphasis on the high rates of resistance to penicillin and macrolides in Streptococcus oralis. J Antimicrob Chemother 1998; 41: 621-627.

18. Kuriyama T, Nakagawa K, Karasawa T, Saiki Y, Yamamoto $E_{1}$ Nakamura S. Past administration of beta-lactam antibiotics and increase in the emergence of beta-lactamase-producing bacteria in patients with orofacial odontogenic infections. Oral Surg Oral Med Oral Pathol Oral Radiol Endod 2000; 89: 186-192.

19. Mainjot A, D'Hoore W, Vanheusden A, Van Nieuwenhuysen J P. Antibiotic prescribing in dental practice in Belgium. Int Endod J 2009; 42: 1112-1117.

20. Patton M O. Qualitative research and evaluation methods. 3rd ed. Thousand Oaks, California: Sage Publishing, Inc, 2002.

21. Braun V, Clarke V. Using thematic analysis in psychology. Qual Res Psychol 2006; 3: 77-101.

22. Boyatzis R E. Transforming qualitative information: thematic analysis and code development. Thousand Oaks, California: Sage Publishing, Inc, 1998.

23. NVivo qualitative data analysis software. 8th ed. OSR International Pty Ltd, 2008.

24. Morse J M. The significance of saturation. Qual Health Res 1995: 5: 147-149.

25. Statistics for Wales. Welsh Index of Multiple Deprivation (WIMD). Cardiff: Welsh Government, 2011. Online information available at http:// wales.gov.uk/statistics-and-research/welshindex-multiple-deprivation/?lang=en\# | statistics-and-research/welsh-index-multipledeprivation/?lang=en (accessed March 2014).

26. Palmer N A, Pealing R, Ireland R S, Martin MV. A study of therapeutic antibiotic prescribing in National Health Service general dental practice in England. Br Dent J 2000; 188: 554-558.

27. Chate R A, White S, Hale L R et al. The impact of clinical audit on antibiotic prescribing in general dental practice. Br Dent J 2006; 201: 635-641.

28. Palmer N A, Dailey Y M, Martin M V. Can audit improve antibiotic prescribing in general dental practice? Br Dent J 2001; 191: 253-255.

29. Seager J M, Howell-Jones R S, Dunstan F D, Lewis $M$ A, Richmond S, Thomas D W. A randomised controlled trial of clinical outreach education to rationalise antibiotic prescribing for acute dental pain in the primary care setting. Br Dent J 2006; 201: 217-222. 